\title{
Dynamics Analysis for Polymer-fiber Composites Based on Shear-ellipsoidal Extensional Force Field
}

\author{
Zan Huang \\ School of Marine engineering, Guangzhou Maritime Institute, Guangzhou 510725, China \\ zhuanggz@aliyun.com
}

Keywords: numerical simulation; orientation distribution; fibers suspensions; shear-ellipsoidal extensional force field

\begin{abstract}
An orientation distribution function is applied to describe the orientation distribution of polymer-fibers composites in shear-ellipsoidal extensional flow. And a mathematical model is established to simulate orientation distribution in this flow. Furthermore, the analytical solutions of differential equations on plant fibers orientation can be acquired in complex flow field. Therefore, analytical solution of differential equation on plant fiber orientation distribution is obtained.
\end{abstract}

\section{Introduction}

In recent years, fibers suspensions of polymer-fiber composites are attracted increasingly in many science and engineering fields, such materials science, paper-making industry, composites material production and so on. Furthermore, the orientation distribution and behavior of fibers is a hot issue increasingly in material processes, such as extrusion, injection, and compression molding. The reason of this phenomenon is the orientation distribution of fibers determines largely the mechanical properties of polymer-fiber composites.

There are some literatures dealing with the evolution of fiber orientation by experimental, numerical and analytical methods for decades. Lin et al. [1] obtained fiber orientation distribution in round turbulent jet of fiber suspension by numerical simulation methods. Najam [2] used a novel methodology in formulating a closure by employing an artificial neural network (ANN) to obtain model of fiber orientation in short fiber suspensions. Luo et al. [3-4] investigate the orientation distribution of fiber suspensions based on planar extensional force field and shear-uniaxial extensional flow. Niskanen H. et al. [5] used flexible wood fibers in dilute suspension to investigate the development of fiber orientation distribution in a plane contracting channel flow by experiments and modeling. Parsheh et al. [6] investigated the influence of shape of planar contractions on the orientation distribution of stiff fibers suspended in turbulent flow. The above research has focused mainly in shear flow, however, few articles studied extensional flow with fiber suspensions.

The objective in this article is to investigate the orientation distribution of fiber suspensions based on shear-ellipsoidal extensional force field.

\section{Mathematical Model}

Jeffery[7] has developed firstily the evolution equation of $P$ for a single fiber immersed in a Newtonian flow without external torque. Where, the expression was shown below:

$\dot{P}=W \cdot P+\lambda(E \cdot P-E: P P P)$.

Where, $\lambda=\left(\beta^{2}-1\right) /\left(\beta^{2}+1\right)$ is the fiber shape factor, $W$ is vorticity tensor of fiber, $E$ is deformation rate of fiber, $\beta=L / d$ is the fiber aspect ratio. Assuming the fiber internal influence is neglected and the fiber has free orientation in initial conditions[8]. The orientation equation with three dimension can be simplified in this condition:

$$
\psi(P, t)=\frac{1}{4 \pi}\left(\Delta^{T} \cdot \Delta: P P\right)^{-\frac{3}{2}} .
$$

The velocity gradient tensor $\nabla v$, vorticity tensor $W$ and rate of deformation tensor of fiber $E$ can be written as: 


$$
\nabla v=\left(\begin{array}{ccc}
2 \dot{\varepsilon} & \dot{\gamma} & 0 \\
\dot{\gamma} & \dot{\varepsilon} & 0 \\
0 & 0 & -3 \dot{\varepsilon}
\end{array}\right), \quad E=\left(\begin{array}{ccc}
2 \dot{\varepsilon} & \dot{\gamma} & 0 \\
\dot{\gamma} & \dot{\varepsilon} & 0 \\
0 & 0 & -3 \dot{\varepsilon}
\end{array}\right), W=\left[\begin{array}{ccc}
0 & 0 & 0 \\
0 & 0 & 0 \\
0 & 0 & 0
\end{array}\right] \text {. }
$$

Where, $v$ is flow velocity, $\dot{\gamma}$ is scalar rate of shear deformation and $\dot{\varepsilon}$ is scalar rate of extensional deformation. Eq.(3c) can be split into two tensor below:

$$
E=\dot{\varepsilon}\left(\begin{array}{ccc}
2 & 0 & 0 \\
0 & 1 & 0 \\
0 & 0 & -3
\end{array}\right)+\dot{\gamma}\left(\begin{array}{ccc}
0 & 1 & 0 \\
1 & 0 & 0 \\
0 & 0 & 0
\end{array}\right)=E^{\prime}+E^{\prime \prime}
$$

Therefore, displacement tensor $\Delta_{i j}$ can be expressed below:

$$
\frac{\mathrm{d} \Delta_{i j}}{\mathrm{~d} \hat{t}}=-\Delta_{i k}^{\prime} E_{k j}^{\prime}-\Delta_{i k}^{\prime \prime} E_{k j}^{\prime \prime} \text {. }
$$

Through Eq.(5) can be shown:

$\frac{\mathrm{d} \Delta_{11}^{\prime}}{\mathrm{d} \hat{t}}=-\left(\Delta_{11}^{\prime} E_{11}^{\prime}+\Delta_{12}^{\prime} E_{21}^{\prime}+\Delta_{31}^{\prime} E_{31}^{\prime}\right)=-2 \Delta_{11}^{\prime}$

$$
\Delta_{11}^{\prime}=e^{-2 t} \text {. }
$$

The other components of $\Delta^{\prime}$ can be acquired by the above similar method. Therefore, deformation tensor $\Delta^{\prime}$ can be shown below:

$$
\Delta^{\prime}=\left(\begin{array}{ccc}
e^{-2 t} & 0 & 0 \\
0 & e^{-t} & 0 \\
0 & 0 & e^{3 t}
\end{array}\right)
$$

Similarly, Through Eq.(7) can be also shown:

$$
\begin{aligned}
& \frac{\mathrm{d} \Delta_{11}^{\prime \prime}}{\mathrm{d} \hat{t}}=-\left(\Delta_{11}^{\prime \prime} E_{11}^{\prime \prime}+\Delta_{12}^{\prime \prime} E_{21}^{\prime \prime}+\Delta_{31}^{\prime \prime} E_{31}^{\prime \prime}\right)=0, \quad \Delta_{11}^{\prime \prime}=1 . \\
& \frac{\mathrm{d} \Delta_{12}^{\prime \prime}}{\mathrm{d} \hat{t}}=-\left(\Delta_{11}^{\prime \prime} E_{12}^{\prime \prime}+\Delta_{12}^{\prime \prime} E_{22}^{\prime \prime}+\Delta_{13}^{\prime \prime} E_{32}^{\prime \prime}\right)=-\dot{\gamma}, \quad \Delta_{12}^{\prime \prime}=-\dot{\gamma} T .
\end{aligned}
$$

The other components of $\Delta^{\prime \prime}$ can be acquired by the above similar method. Therefore, deformation tensor $\Delta^{\prime \prime}$ can be shown below:

$$
\Delta^{\prime \prime}=\left(\begin{array}{ccc}
1 & -j T & 0 \\
-j T & 1 & 0 \\
0 & 0 & 1
\end{array}\right)
$$

The last deformation tensor $\Delta$ can be shown below:

$$
\Delta=\lambda_{1} \Delta^{\prime}+\lambda_{2} \Delta^{\prime \prime}=\left(\begin{array}{ccc}
0.8 e^{-2 t}+0.2 & -0.2 \dot{\gamma} T & 0 \\
-0.2 j T & 0.8 e^{-t}+0.2 & 0 \\
0 & 0 & 0.8 e^{3 t}+0.2
\end{array}\right) .
$$

Where, $\lambda_{1}=0.8$ and $\lambda_{2}=0.2$ is the weight parameter, respectively.

Therefore:

$$
\Delta^{T} \Delta=\left(\begin{array}{ccc}
0.64 e^{-4 t}+0.32 e^{-2 t}+0.04 t^{2}+0.04 & -0.16 t e^{-2 t}-0.16 t e^{-t}-0.08 t & 0 \\
-0.16 t e^{-2 t}-0.16 t e^{-t}-0.08 t & 0.64 e^{-2 t}+0.32 e^{-t}+0.04 t^{2}+0.04 & 0 \\
0 & 0 & 0.64 e^{6 t}+0.32 e^{3 t}+0.04
\end{array}\right)
$$

Eq.(11) are substituted into Eq.(2) and shown below:

$$
\psi(P, t)=\frac{1}{4 \pi}\left(\Delta^{T} \cdot \Delta: P P\right)^{-\frac{3}{2}}
$$




$$
\begin{gathered}
\quad=\frac{1}{4 \pi}\left[\left(\Delta_{k} \Delta_{k j} e e\right): P e_{s} P_{s} e_{t}\right]^{-\frac{3}{2}} \\
=\frac{1}{4 \pi}\left(\Delta_{k} \Delta_{k s} P_{s} P_{t}\right)^{\frac{3}{2}} \\
=\frac{1}{4 \pi}\left(\Delta_{11}^{T} \Delta_{11} P_{1} P_{1}+\Delta_{22}^{T} P_{2} P_{2}+\Delta_{33}^{T} \Delta_{33} P_{3} P_{3}+\Delta_{12}^{T} \Delta_{12} P_{1} P_{2}+\Delta_{21}^{T} \Delta_{21} P P_{1}\right)^{-\frac{3}{2}} \\
=\frac{1}{4 \pi}\left[\left(0.64 e^{-4 t}+0.32 e^{-2 t}+0.04 t^{2}+0.04\right) \sin ^{2} \theta \cos ^{2} \phi\right. \\
+\left(0.64 e^{-2 t}+0.32 e^{-t}+0.04 t^{2}+0.04\right) \sin ^{2} \theta \sin ^{2} \phi+\left(0.64 e^{6 t}+0.32 e^{3 t}+0.04\right) \cos ^{2} \theta \\
\left.+2\left(-0.16 t e^{-2 t}-0.16 t e^{-t}-0.08 t\right) \sin ^{2} \theta \sin 2 \phi\right]^{-\frac{3}{2}} .
\end{gathered}
$$

Where, $t=\dot{\gamma} T_{0}$ expresses dimensionless cumulant along with time, $T_{0}$ means current time.

\section{Results and Discussion}

Interval $\theta \in(0, \pi)$ and interval $\phi \in(0,2 \pi)$ are divided 100 nodes respectively. According to Eq.(13) of analytical solution of orientation distribution function of fiber in shear-ellipsoidal extensional flow, several pictures can be shown by numerical simulation in Fig. 1 and Fig. 2.

The evolution characteristics of the fiber dimensional orientation each extensional segment can be shown obviously from $T=0.1$ to $T=4.0$ in Fig. 1. The orientation of fiber is clutter at initial moments. However, there are more fibers at the directions of $(\theta, \phi)=\left(\frac{\pi}{2}, 0\right),(\theta, \phi)=\left(\frac{\pi}{2}, \pi\right),(\theta, \phi)=(\pi / 2,3 \pi / 2)$ and $(\theta, \phi)=\left(\frac{\pi}{2}, 2 \pi\right)$. Furthermore, the plant fibers have a tendency obviously to gather in the three directions with the passage of time in the flow field. In general, there are four peaks. There is an obvious gather trend at the above three directions along with time increasingly. Therefore, the orientation of fiber at the above three directions is higher greatly than other directions. Furthermore, the extensional deformation accumulates and the orientation of fiber is more and more obvious along with time increasingly. Therefore, the slope of orientation is more and more sharp and the maximum value of orientation distribution function $\psi(\theta, \phi, t)$ is close to the position of $\phi=0$.
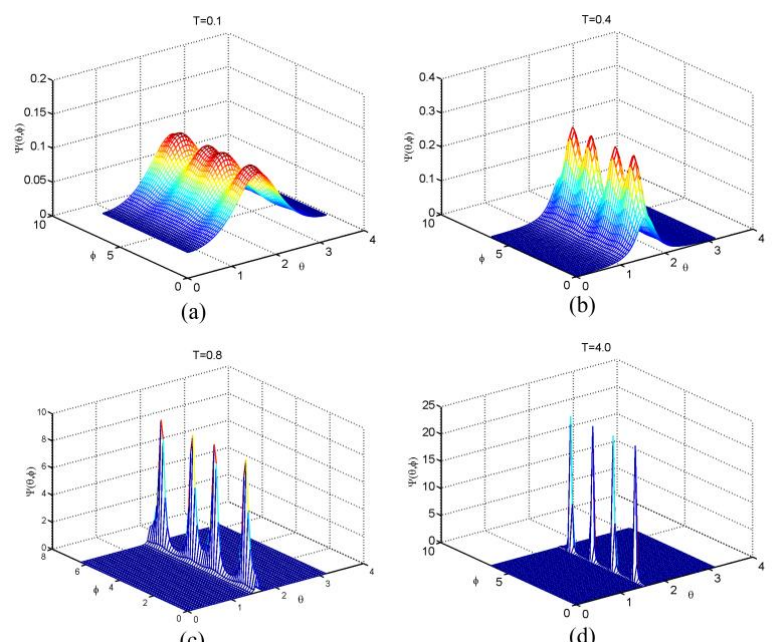

(c)

Fig. 1 The fiber orientation distribution function evolves for shear-ellipsoidal extensional flow field

From figure 2, it can reflect the orientation change characteristics of plant fiber at the surface $\theta=\pi / 2$ clearly. At the beginning, the slope of orientation distribution of plant fiber is slower and wider, it also means that the orientation distribution of plant fiber is relatively clutter. The slope of fiber orientation distribution is relatively gentle and the fiber distribution is relatively clutter in the 
short time interval. The orientation of fiber at the direction of $\phi=\pi / 2,3 \pi / 2$ achieves maximum and minimum values respectively. However, gathered trend of fiber can be seen clearly, orientation is more and more obvious and the degree of orientation is sharp increasingly at angle of orientation $\phi=\pi / 2,3 \pi / 2$ along with increasing time. Finally, fiber orientation exists only at angle of orientation $\phi=\pi / 2,3 \pi / 2$. In fact, fiber orientation is along with the direction of extend, due to fiber has no difference between head and tail. However, due to the gradient of fiber orientation is very cliffy, the step length $\delta \theta, \delta \phi$ has great influence to the result of accuracy. Furthermore, it can be seen gathered trend at $\phi=\pi / 2,3 \pi / 2$ and the orientation changes obviously increasingly, the degree of orientation is becoming more and more sharp.

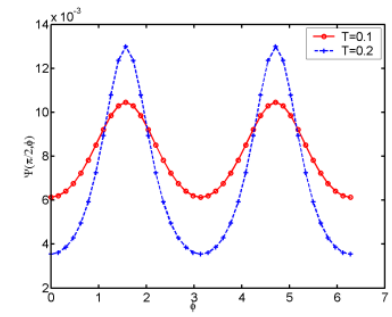

(a)

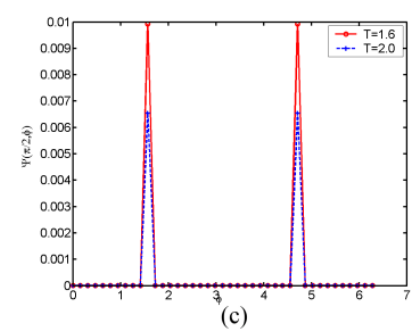

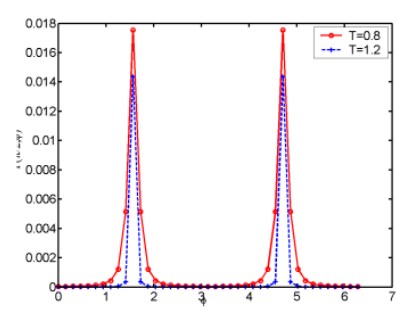

(b)

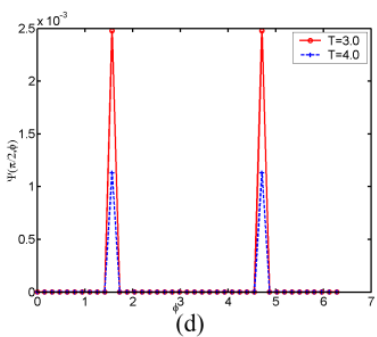

Fig.2 The fiber orientation distribution changing for shear-ellipsoidal extensional flow field at $\theta=\pi / 2$

\section{Conclusions}

The greater of tensile deformation rate, the shorter of period of motion; the bigger of length to diameter ratio,the longer of period of motion. The motion of fiber is not the uniform periodic motion, but it is sometimes fast and sometimes slow. The value of fiber orientation distribution function is relevant with initial angle and cumulative deformation in shear-ellipsoidal extensional flow field. The numerical distribution of fiber orientation distribution function has great variation in shear-ellipsoidal extensional flow field. When extensional deformation rate is small, its numerical distribution is wide. However, the orientation distribution of fiber is more and more narrow along with extensional deformation increasingly. It tends to the direction of extend flow gradually.

\section{Acknowledgements}

The authors wish to acknowledge the Guangzhou Science and Technology Plan Project (201541) and the "Innovation and Strengthen University" Project of Guangzhou Maritime Institute (A330106, B510647)for financial support.

\section{References}

[1] Lin J. Z.; Liang X. Y.; Zhang S. L. Chemical Engineering Research and Design, 2012, 90(6): 766-775.

[2] Najam ul Qadir, David A. Jack. Modeling fiber orientation in short fiber suspensions using the 
neural network-based orthotropic closure [J]. Composites: Part A, 2009, 40, 1524-1533.

[3] Luo P F, Huang Z. Numerical Simulation of Orientation Distribution on Fibers Suspensions in Planar Extensional Field[J]. Applied Mechanics and Materials, 2013, 395: 1174-1178.

[4] Luo P F. Dynamics Analysis of Orientation Distribution of Fiber Suspensions in Shear-extensional Flow[J]. Applied Mechanics and Materials, 2015,709: 168-171.

[5] Niskanen H., Eloranta H., Tuomela H., Hämälä;nen J. On the orientation probability distribution of flexible fibers in a contracting channel flow [J]. International Journal of Multiphase Flow, 2011, 37, 336-345.

[6] Parsheh M.; Brown M. L.; Aidun C. K. International Journal of Multiphase Flow, 2006, 32(12): 1354-1369.

[7] Jeffery G. B. Proceedings of Royal Society of London, 1922, 102(715): 161-179.

[8] Bees M. A.; Hill N. A.; Pedley T. J. Journal of Mathematical Biology, 1998, 36(3):269-298. 